bewiesene Tatsache, daß die Dicke der elektrischen Doppelschicht die Molekulardimensionen weit übertrifft.
Herrn Prof. J. J. Boguski möchte ich für bereitwilliges Entgegenkommen bei meiner Arbeit meinen besten Dank aussprechen.

\title{
Die Untersuchung der Nitrozellulosen im Polarisationsmikroskop.
}

\author{
Von Hans Ambronn (Jena). \\ (Mitteilung aus dem Institut für Mikroskopie an der Universität Jena.)
}

(Eingeg. an 10. September 1913)

Schon vor 50 Jahren hatte C. Näge li ${ }^{i}$ ) beobachtet, daß das optische Verhalten der Baumwolle bei ihrer Umwandlung in Schiebwolle wesentliche Veränderungen erfährt. Während die Gestalt der Fasern, also ihre äuBere Form, bei der Einwirkung der Nitriersäuren erhalten bleibt, wird die Stärke und auch der Charakter der Doppelbrechung durch die Nitrierung beträchtiich beeinllußt. Schon Nägeli stellte fest, daf schwach nitrierte Baumwolle etwas geringere Doppelbrechung zeigt als die reine, aber noch de ns elben Charakter besitzt, daf dagegen die stärker nitrierte Faser den $4 \mathrm{~m}$ gekehrten Charakter der Doppelbrechung erkennen läßt.

Spätere Beobachter scheinen diese wichtige Tatsache gar nicht recht beachtet $z u$ haben. Die in der Literatur vorhandenen Angaben beziehen sich fast stets nur auf die Stärke der Doppelbrechung, d. h. auf die zwischen ge kreuzten Nikols auftretenden Interferenzfarben. lassen aber den verschiedenen Charakter in der Doppelbrechung der einzelnen Nitrierungsstufen ganz unbeachtet. Selbst H. de Chard o n ne ${ }^{2}$ ) und G. Lung ${ }^{3}$ ), die sich mit dem optischen Verhalten der Fasern noch am meisten beschäftigt haben, sprechen iiberhaupt nicht von der Umketirung des Vorzeichens der Doppel. brechung.

Im folgenden soll aber eine genauere Untersuchung berichtet werden, die sich mit der Frage beschäftigte, welche Beziehungen zwischen optischem Verhalten und Stickstoffgehalt der Fasern bestehen. Díe vollständige Zusammenstellung der Literatur, sowie die ausführliche Beschreibung der angestellten Versuche wird in meiner dernuächst erscheinenden. Inaugural-

1) C. Nägeli, Beobachtungen über das Verbalten des polarisierten Lichtes gegen pflanzliche Organismen; Ber. d. Bayer. Akad. 1862, 1, 307.

2) H. de Chardonnet. Compt. rend. 106,. 633 (1888); 145, 115 (1.907).

3) G. Lunge u. Weintraub, Beiträge zur Kenntnis der Nitrozellulose; Zeitschr. f. angew. Chem. 1899, 441 ff. G. Lunge u. Bebie, Nitrozelutosen; Zeitschr. f. angew. Chem. 1901, 561 ff. dissertation gegeben werden. Hier seien nur die wichtigsten Resultate mitgeteilt.

I.

Beobachtungen an nitrierten Baumwollen.

Als Beobachtungsmaterial benutzten wir zunächst Brumwollen, Kollodiumwollen und Schießwollen, die Herr Geheimrat Prof. Dr. Ost in Hamrover uns freundlichst aus seiner Sammlung zur Verfügung stellte. Durch seine Vermittlung erhielten wir weitere Proben mit Angabe des N-Gehaltes aus der Pulver- tnd Sprengstofiabrik Wolff \& Co. in Walsrode und von der Rheinisch-Westfälischen Sprengstoff-Altiengesellschaft Pulverfabrik Troisdorf ${ }^{4}$ ).

Eine Untersuchung mit dem gewöhnlichen Mikroskop gibt nach Th. Hartig ${ }^{5}$ ) keinen sicheren Anhaltspunkt zur Unterscheidung von reiner und nitrierter Baumwolle. Beobachtet man dagegen mit einem Polarisationsmikroskop, so wird man Nägeli's Resultate bestätigt finden.

Reine Baumwolle ist stark doppelbrechend und zeigt zwischen gekreuzten Nikols verschiedene Interferenzfarben erster Ordnung; denn je nach der Dicke der einzelnen Fasern zeigen sich Grau, Weiß, Orange, Rot und alle Uebergänge in demselben Präparat. Hauptsächlich war lebhaftes $\mathrm{Gelb}^{6}$ ) zu beobachten. Die Fasern der reinen Baumwolle sind in bezug auf ihre Längsrichtung positiv doppelbrechend.

Sogenannte Kollodiumwollen, d. h. schwach nitrierte Baumwollen, zeigen ebenfalls Farben erster Ordnurg, besonders treten Lavendelgrau

*) Auch an dieser Stelle möchte ich vor allem Hem Seheimrat Prof. Dr. Ost und den Leitungen oben genannter Fabriken für die freundliche Unterstuitzung danken; die uns durch das Ueberlassen des Materials zuteil wurde. Die einzelnen Proben werden nach des Fabribbezeichnungen zitiert.

5) Th. Hartig, Untersuchungen über den Bestand und die Wirkung der explosiven Baumwolle mit besonderer Berücksichtigung des mikroskopisch Nachweisbaren vor, während und nach der Explosion (1847).

b) Die Interferenzfarben sind nach der Tabelle von G. Quincke zitiert; Pogg. Ann. 129, 180 (1866). 
bis Graublau hervor. Sie sind positiv doppelbrechend wie reine Baumwolle; jedoch ist thre Anisotropie erheblich schwächer.

Höher nitrierte Schießwolle dagegen leuchtet im polarisierten Licht blat auf. Die Doppelbrechung ist schwach und in bezug auf die Längsrichtung der Faser negativ.

Die. reine Baumwolle und die SchieBwolle haben mithin entgegengesetzten Charakter der Doppelbrechung. Nun liegt die Vermutung nahe, daß zwischen beiden Stadien Uebergänge liegen, die wir mit Hilfe verschiedener Nitrierungsstufen festzustellen suchten. Zu diesem $Z$ wecke lịeferten uns die schon erwähnten Fabriken das Material. Es waren die gebräuchlichsten Sorten mit Angabe des N-Gehaltes.

Die Fabrik in. Walsrode stellte uns fünf Proben gemahlener Wolle zur Verfügung, die folgendes Resultat ergaben:

Probe I hat 10,8 Proz. N. Die Eigenfarbe ist grau. Es liegt positive Doppelbrechung vor.

Probe II hat 11,7 Proz. N. Die Fasern sind nur schwach anisotrop; einige hellen das $\mathrm{Ge}$ sichtsfeld gar nicht auf. Die Eigenfarbe der doppelbrechenden Farben ist grau; sie sind positiv doppelbrechend.

Probe III hat 12,5 Proz. N. Die Fasern leuchten zwischen gekreuzten Nikols schwach bläulich auf. Diese Probe hat bereits negativen Charakter, der Anisotropie.

Probe IV enthält 12,8 Proz. N. Die Fasern zeigen blaue Eigenfarbe im polarisierten Licht und sind negativ doppelbrechend.

Probe V enthält 13,2 Proz. N. Sie ist stark doppelbrechend und leuchtet $z$ wischen gekreuzten Nikols blau auf. Der Charakter der Doppelbrechung ist negativ.

Aehnliche Resultate ergaben die Proben von Troisdorf:

PC 245 ist nach Angabe der Pabrik Zelluloidpapier mit 10,54 Proz: N. Diese Probe ist aus Baumwollpapier hergestellt und dient zur Zelluloidbereitung. Die Fasern sind positiv doppelbrechend. Zwischen gekreuzten Nikols zeigen sie meist das klarere Grau.

TII 254 ist Zelluloid-Kollodiumwolle mit 10,97 Proz. N. Sie dient gleichfalls zur Zelluloidbereitung und ist wie die folgenden Proben aus Baumwolle gewonnen. Sie ist nur schwach doppelbrechend, und zwar positiv. Die Fasern leuchten nur schwach graubiau auf.

H131 ist Zelluloid-Kollodiumwolle mit 12,13 Proz. N, die zur Zelluloidherstellung benutzt wird. Sie ist nur schwach anisotrop; die Eigen- farbe ist bläulich, der Charakter der Doppelbrechung negativ.

H 176 ist Dynamit-Kollodiumwolle mit 12,33 Proz. N. Sie dient zur Herstellung von Dynamit. Sie ist schwach anisotrop. Zwischen gekreuzten Nikols leuchtet sie schwach bläulich auf; es liegt negative Doppelbrechung vor.

H 50 ist SchieBwolle mit 12,80 Proz. N. Die Anisotropie ist wieder stätker. Die Fasern leuchten zwischen gekreuzten Nikols blau auf. Der Charakter läßt sich als negativ feststellen.

H 146 ist hochprozentige SchieBwolle mit 13,22 Proz. N. Die Probe ist stark anisotrop. Im polarisierten Lichte leuchten die Fasern blaw. auf. Sie sind negativ doppelbrechend.

Es sinktalso beisteigendem Stickstoffgehalt dieDoppelbrechung, geht durch Null hindurch und steigt dann wieder. Bei niedrigem Stickstoffgehaltist siepositivund bei höherem negativ. Aus diesen Versuchsergebnissen läBt sich weiter folgern, daß bei dem NitrierungsprozeB der Baumwolle der Charakter der Doppelbrechung sich nicht plötzlich umkenrt. Er wandert vielmehr allmählich bei steigendem Stickstoffgehalt von positiv durch Null nach negativ.

Genauere quantitative Bestimmungen der Stärke der Doppelbrechung in den einzelnen Fällen konnten an dem vorliegenden Material nicht vorgenommen werden, da die Fasern durch das Mahlen stark zerrissen waren. Es lag deshalb die Frage nahe, ob die langeren unzerstörten Fasern gleichmäßig nitriert sind oder in ihrer Längserstreckung verschiedene Nitrierungsstufen aufweisen. Solche Proben stellte uns die Pulverfabrik in Walsrode zur Verfügung. Leider war darunter keine Probe, die weniger als 11,8 Proz. $\mathrm{N}$ aufwies. Bel diesen Proben wurde auch die Phasendifferenz mit Hilfe des Quarzkeil-Kompensator-Okulars nach H.S i e d e $n$ to $\mathrm{p}^{7}$ ) bestimmt. Als Lichtquelle diente die Quecksilberbogenlampe mit Lichtfiltern nach $A$. Köhle ${ }^{8}$ ), sogenannte HagehLampe von $C_{\text {. }}$ Zeiss in Jena. Die Filter gestatten monochromatisches Licht der Wellenlängen 435,546 und $579 \mu \mu$ von genügender Intensität herzustellen, so daB die Verschiebung der dunklen Streifen des Quarzkeils leicht abgelesen werden kann. Für die Phasendifferenz d

7) H. Siedentopt, Gebranchsanweisung von C. Zeiss; Mikro 212.

8) A. Kö hler, Ueber die Verwendung des Quecksilberlichtes fir mikioskopische Arbeiten. Zeitschr.f. wiss. Mikrosk. u mikr. Technik 27, 329 - 335 (1910). 
besteht bekanntlich die Formel: $\delta=\frac{d\left(n_{2}-n_{1}\right)}{\lambda}$ worin $\mathrm{d}$ die Dicke der Faser, $\mathrm{n}_{\mathrm{a}}-\mathrm{n}_{1}$ die Stärke der Doppelbrechung und $\lambda$ die Wellenlănge des zur Beleuchtung benutzten Lichtes bedeutet. Da zur Untersuchung immer möglichst gleichdicke Fasern ausgewählt wurden, so darf $\mathrm{d}$ als konstant angesehen werden, so daf 3 also die Resultate untereinander direkt vergleichbar sind.

Die Proben ergaben folgendes Resultat:

Schiebwolle mit 13,2 Proz. N. Die Doppelbrechung ist ziemlich stark; die Fasern sind gleichmäbig nitriert. Sie leuchten zwischen gekreuzten Nikols blau auf. Es liegt negative Doppelbrechung vor.

Kollodiumwolle mit 12,2 Proz. N. Diese Probe ist ungleichmäBig nitriert, soweit man dies aus dem optischen Verhalten schliehien kann. Es wurden immer nur Fasern ausgesucht, die ein gleichmäBiges Bild gaben. Sie leuchten im polarisierten Licht blau anf und sind negativ doppelbrechend.

BN-Wolle für technische Zwecke hat 11,9 Proz. N. Sie ist gleichmabig nitriert. Zwischen gekreuzten Nikols leuchtet sie schwach blau auf; sle ist schwach negativ doppelbrechend.

B-Wolle für technische Z wecke hat 1 1,8 Proz.N. Die Fasern leuchten zum gröBten Teil zwischen gekreuzten Nikols überhaupt nicht mehr deutlich auf; sie sind für eine Farbe wenigstens isotrop.

Es konnte an diesen Proben festgestellt werden, dab die Fasern in ihrer ganzen Lange gleichmäbig nitriert waren, aber manchmal, wie bei der Kollodiumwolle, nicht alle Fasern im selben Grade. In diesem Fall finden sich neben höher nitrierten solche mit niedrigerem Stickstoffgehalt. Die Nitrozellulose andert den Charakter ihrer Doppelbrechung bei etwa 11,8 Proz. N. Als Mittel der Phasendifferenzen fanden wir aus je acht Bestimmungen folgende Werte in Wellenlängen:

\begin{tabular}{c|c|c|c|c}
\hline Probe & Proz.N & $435 \mu \mu$ & $546 \mu \mu$ & $579 \mu \mu$ \\
\hline SchleBwolle : & 13,2 & $-0,20$ & 0,15 & $-0,11$ \\
Kollodiumwolle & 12,2 & $-0,08$ & $-0,05$ & $-0,03$ \\
BN-Wolle : & 11,9 & $-0,05$ & 0 & $+0,01$ \\
B-Wolle . : & 11,8 & 0 & $+0,05$ & $+0,08$
\end{tabular}

Hieraus geht hervor, daß die Phasendifferenz bei hochnitrierter Baumwolle für alle drei Wellenlängen negativ ist. Bei der Stufe mit 11,8 Proz. N ist sie schon für $435 \mu \mu$ null, für die beiden anderen aber positiv.
II.

Beobachtungen an nitrierter Ramie.

Die bisher erwähnten Proben bestanden alle aus Baumwolle. Nun sind sowohl die reinen, wie auch die nitrierten. Baumwollfasern wenig für eine solche systematische Untersuchung geeignet. Dénn die Torsionen, die diese Fasern fast stets besitzen, beeinträchtigen eine genaue Beobachtung sehr. Eine Faser, die diese Nachteile nicht hat und sich deshalb $z u$ derartigen Versuchen sehr eignet, ist die unter dem Namen Ramie bekannte Bastfaser der Boehmeria tenacissima Gaud.

Zunächst nitrierten wir die gut gereinigten Ramiefasern mit reiner Salpetersăure ohne $\mathrm{Zu}$ satz von Schwefelsäure. Hierbei läft sich der Vorgang unter dem Mikroskop leicht verfolgen. Man beobachtet die Faser im Wasser und findet, dah sie stark positiv doppelbrechend ist; sie zeigt hohe Interferenzfarben bis Rot zweiter Ordnung. Nun saugt man das Wasser mit Fliebpapier ab und läbt von einer Seite die Salpetersäure zulaufen. pie Faser quillt etwas auf und zeigt eine einheitlichere färbung als zuvor. Sie leuchtet schwach blau auf; die Doppelbrechung ist gesunken. Diese Ansicht gewährt die Paser auf der Seite, von der die Säure zuflo8, während der übrige Teil noch das Bild der reinen Ramie gibt, weil hier noch keine Einwirkung der Säure stattgefunden hat. Erstere Stelle ist bereits negativ doppelbrechend. Je weiter die Salpetersäure eindringt, um so gröBere Partien der Faser werden negativ doppelbrechend, indem der Charakter der Doppelbrechung von positiv durch Null nach negativ wandert. Da die konzentrierte Säure die Paser auflöst, ist eine längere Beobachtung nicht möglich. Versuchten wir aber der zerstörenden. Wirkung durch Verdinnen Einhalt zu tun, so wurde die Doppelbrechung wieder positiv. Auch mit Gemischen aus Salpeter- und Schwefelsäure nitrierten wir die Fasern und erhielten das gleiche Resultat. Die anfangs vorhandene starke positive Doppelbrechung der reinen Ramiefaser wird während der fortschreitenden Nitrierung immer schwäcberund geht schlieblichdurch Null in die negative Doppelbrechung der hochnitrierten Faser über.

Um zu den weiteren Versuchen, vor allem zu den quantitativen Bestimmungen der durch die Doppelbrechung entstehenden Phasendifferenzen, ein möglichst gleichmäßfiges Material in größerer Menge zur Verfïgung zu haben, und um auperdem auch die Produkte prïfen zu können, die 
bei den üblichen in der Gromindustrie angewandten Methoden sich ergeben, wandten wir uns an die Pulverfabrik Troisdorf, die uns bereitwilligst nitrierte Ramiefasern in folgenden Proben herstellte.

Die Fasern sind in ihrer ganzen Länge gleichmäBig nitriert und in ihrer auberen Form von gewöhnlichen Fasern kaum zu unterscheiden, nur das Lumen hat einen etwas geringeren Durchmesser $\%$. Die Prüfung des optischen Verhaltens ergab folgende Resultate:

Probe I mit 13,16 Proz. $N$ leuchtet zwischen gekreuzten Nikols blau auf und erweist sich als optisch negativ.

Probe li mit 12,85 Proz. N leuchtet blabblau auf und ist ebenfalls negativ doppelbrechend.

Probe III mit 11,88 Proz. $\mathrm{N}$ ist nur ganz schwach anisotrop, ja fast isotrop. Die Eigenfarbe ist je nach der Wellenlänge, für die Isotropie besteht, verschieden: gelb, blau, violett. Auch der Charakter der Doppelbrechung ist infolgedessen je nach der Fäbe verschieden.

Probe IV hat 10,55 Proz. N; die Fasern sind stark doppelbrechend, und zwar positiv. lhre Eigenfarbe zwischen gekreuaten Nikols ist gelblichweib.

Bei diesen Proben stellten wir wieder die Phasendifferenzen für die Wellenlängen 435, 546 und $579 \mu \mu$ mit der Hageh-Lampe fest. Außerdem benutzten wir noch eine gute Rotglasplatte, die Licht von ca. $650 \mu \mu$ Wellenlänge durchlaßt. Als Lichtquelle hierfür diente eine Nernstlampe nach $H$. Siedentopf. Die Messungen ergaben im Mittel aus je zehn Ablesungen in Wellenlängen ausgedrückt:

\begin{tabular}{|c|c|c|c|c|c|}
\hline Probe & $35 \mu \mu$ & $546 u \mu$ & $579 \mu \mu$ & $650 \mu \mu$ & $\nu$ \\
\hline a & & & & & \\
\hline & $-0,5$ & $-0,32$ & $-0,31$ & $-0,23$ & $+1,46$ \\
\hline & $-0,32$ & $-0,20$ & $-0,17$ & $-0,12$ & $+1,86$ \\
\hline $\begin{array}{l}\text { Rami } \\
11,8\end{array}$ & $-0,12$ & $-0,08$ & 0 & $+0,02$ & -5 \\
\hline $\begin{array}{r}\text { Rami } \\
10,5\end{array}$ & +0 & $+0,32$ & $+0,31$ & $+0,30$ & $+0,75$ \\
\hline aine Ramie & +28 & $+2,2$ & $+2 ; 07$ & $+1,91$ & $+0,98$ \\
\hline
\end{tabular}

9) Es möge hier nur ganz kurz darduf hingewiesen werden, daß auch die Zugiestigkeit der Fasern durch die Nitrierung nicht unbetrẳchtlich gesindert wird. Die Festlgkelt der hoch nitrierten probe von 13,16 Proz. $N$ ist nur etwa halb so grobals die der reinen Ramlefasern.

10) Fur dle Bestimmung dieser Werte wurden die Fasern gewählt, die mattblaue Eigenfarbe zeigten, bei denen also fïr gelbes Licht annahernd Isotropie bestand,
Wie aus der Tabelle zu ersehen ist, nimmt hier die Phasendifferenz mit der Größe der Wellenlänge ab. Dies ist jedoch keineswegs allgemein der Fall, wie auch aus den folgenden Tabellen hervorgeht, in denen für eine ganze Reihe von Stufen das Ungekehrte gilt. Es rührt dies daher, dab die sogenannte Dispersion der Doppelbrechung

$$
v=\frac{\left(n_{2}-n_{1}\right) 435 \mu \mu}{\left(n_{2}-n_{1}\right)_{650 \mu \mu}}
$$

gerade in den Uebergangsstadien starke Ver. änderung zeigt. In der letzten Spalte sind diese Werte für $v$ angegeben. Ferner ist hieraus zu entnehmen, daf nicht für alle Farben gleichzeitig Is otropie eintritt.

III.

\section{Denitrierung.}

Da bel der Nitrierung die Doppelbrechung der Ramie von positiv durch Null nach negativ wandert, war anzunehmen, daB dieser Weg umgekehrt durchlaufen würde, wenn man die $\mathrm{Ni}$ tration der Faser wieder rückgängig machte. Hierzu verwandten wir Ammoniumsulfid, welches. die Fasern in ihrer äuberen Form nicht verändert, sie aber voliständig denitriert. Die allmähliche Denitrierung wurde unter dem Mikroskop in der Weise verfolgt, dab die Faser in Ammoniumsulfid eingebettet und dann der Rand des Deckglüschens mit Paraffin abgeschlossen wurde. Dadurch verzögert sich zwar die Umwandlung ein wenig; man hat aber den Vorteil bequemeren Arbeiters, weil das Denitrierungsmittel nicht verdampft. Durch die Einwirkung des Ammoniumsulfides sinkt die Doppelbrechung der Fasern allmählich auf Null herab, dann steigt sie wieder an und erreicht bald eine gröhere Stärke als zu Anfang des Versuches. Jetzt erweist sie sich aber als positiv und gewinnt nach längerer Einwirkung wieder diejenige Stärke der Doppelbrechung, die der nicht. behandelten Ramie eigentümlich ist. 'Werden die Fasern nach der völligen Denitrierung gut ausgewaschen, so lassen sie sich mit Chlorzinkjodlösung färben. Sowohl aus dem Eintreten dieser Reaktion, wie auch aus der wieder erreichten starken positiven Doppelbrechung darf man den Schluß ziehen, daß nach Beendigurig des Denitrierungsverfahrens reine Ranile vorliegt.

Wir verfolgten die Umwandlung auch quantitativ, indem wir die Phasendifferenzen für ver-

wahrend sie für $435 \mu \mu$ noch negativ, fur $650 \mu \mu$ dagegen schon positiv doppejbrechend waren. 
schiedene Wellenlängen festsiellten und die Werte zu einer Tabelle vereinigten. Es wurde eine bestimmte Stelle einer Faser mit Tusche markiert, so daß man sie während des ganzen Vorgranges, also bei gleicher Dicke, beobachten konnte. Sehr interessant ist die Umwandlung besonders in der Nähe der Isotropie, weswegen hier auch die Ablesungen fïr jede Viertelstunde angegeben sind; für das spätere Stadium genügen längere Zeiten, da diese W.erte ja auch bei den niedrigeren Nitrierungsstufen wiederkehren.

Tabelle der Gangusterschiede bei der Denitrierung von Ramie mit 13,16 Proz. N.

\begin{tabular}{r|c|c|c|c|c}
\hline & $435 \mu \mu$ & $546 \mu \mu$ & $579 \mu \mu$ & $650 \mu \mu$ & $\nu$ \\
\hline 1 & $-0,50$ & $-0,32$ & $-0,31$ & $-0,23$ & $+1,46$ \\
2 & $-0,40$ & $-0,27$ & $-0,25$ & $-0,19$ & $+1,5$ \\
3 & $-0,32$ & $-0,20$ & $-0,19$ & $-0,12$ & $+1,8$ \\
4 & $-0,23$ & $-0,14$ & $-0,13$ & $-0,07$ & +2 \\
5 & $-0,16$ & $-0,10$ & $-0,08$ & $-0,02$ & $+3,5$ \\
6 & $-0,11$ & $-0,06$ & $-0,04$ & 0 & $\infty$ \\
7 & $-0,07$ & $-0,02$ & 0 & $+0,02$ & 2,3 \\
8 & $-0,05$ & 0 & $+0,02$ & $+0,04$ & 0,80 \\
9 & $-0,02$ & $+0,02$ & $+0,05$ & $+0,08$ & $-0,12$ \\
10 & 0 & $+0,06$ & $+0,08$ & $+0,11$ & 0 \\
11 & $+0,01$ & $+0,10$ & $+0,11$ & $+0,15$ & $+0,04$ \\
12 & $+0,03$ & $+0,12$ & $+0,13$ & $+0,18$ & $+0,11$ \\
13 & $+0,07$ & $+0,14$ & $+0,16$ & $+0,20$ & $+0,23$ \\
14 & $+0,14$ & $+0,16$ & $+0,18$ & $+0,23$ & $+0,40$ \\
15 & $+0,26$ & $+0,31$ & $+0,31$ & $+0,30$ & $+0,58$ \\
16 & $+0,50$ & $+0,51$ & $+0,51$ & $+0,40$ & $+0,84$ \\
17 & $+0,80$ & $+0,78$ & $+0,77$ & $+0,61$ & $+0,87$ \\
18 & +1 & $+0,82$ & $+0,81$ & $+0,73$ & $+0,89$ \\
19 & $+1,45$ & $+1,20$ & $+1,12$ & +1 & $+0,95$ \\
20 & $+2,65$ & $+2,30$ & $+2,01$ & $+1,80$ & $+0,99$
\end{tabular}

Die Ablesungen erfolgten bis 5 halbstündlich, bis 11 viertelstündlich, bis 14 halbstíndlich, dann nach je 3 Stunden, von 18 an etwa alle 12 Stunden.

Aus vorstehender Tabelle ersieht man, daB die Isotropie zuerst für rotes, dann für gelbes und zuletzt für blaues Licht eintritt. Die Angabe der Zeit hat keinen absoluten Wert, da die Umwandlung durch öfteres Ennevern des Ammoniumsulfides und gutes Auswaschen variiert werden kann. Parallelversuche, die in größerer Zahl angestellt wurden, ergaben ähnliche Werte. In der letzten Spalte ist wiederum die Dispersion der Doppelbrechung für Blau und Rot verzeichnet; sie steigt von 1,46 bis $+\infty$, kommt dann von $-\infty$, geht durch Null und nähert sich asymptotisch dem Werte 1. In den folgenden Tabellen sind die Werte für die Proben mit 11,88 Proz. $N$ und 10,55 Proz. $N$. angegeben.
Tabelle der Gangunterschiede bei der Denitrierung von Ramie mit 11,88 Proz. N.

\begin{tabular}{|c|c|c|c|c|c|}
\hline & $435 \mu^{\prime \prime \prime}$ & $546 u$ & $579 \mu \mu$ & $650 \mathrm{"I \prime \prime}$ & $\nu$ \\
\hline & & & & & \\
\hline & - 0,12 & $-0,08$ & 0 & $+0,02$ & -5 \\
\hline 2 & $-0,05$ & 0 & 0,02 & $+0,03$ & -1 \\
\hline 3 & 0 & $+0,05$ & $+0,05$ & $+0,09$ & 0 \\
\hline 4 & $+0,09$ & $+0,13$ & 0,15 & $+0,16$ & $+0,4$ \\
\hline 5 & $+0,21$ & $\begin{array}{r}+0.22 \\
+\end{array}$ & $+0,23$ & $+0,23$ & $+0,6$ \\
\hline 6 & $+0,30$ & $+0,30$ & $+0,30$ & $+0,30$ & $+0,65$ \\
\hline 7 & $+0,37$ & $+0,35$ & $+0,35$ & $+0,33$ & $+0,73$ \\
\hline 8 & $+0,41$ & $+0,38$ & $+0,37$ & $+0,35$ & $+0,78$ \\
\hline 9 & $+0,46$ & 0,40 & $+0,39$ & $\begin{array}{r}+0,37 \\
\end{array}$ & $\begin{array}{r}+0,87 \\
\end{array}$ \\
\hline 10 & $+0,51$ & $\begin{array}{r}+0,41 \\
\end{array}$ & $+0,41$ & $\begin{array}{r}+0,38 \\
\end{array}$ & $\begin{array}{r}+0,89 \\
\end{array}$ \\
\hline 11 & $\begin{array}{r}+1,09 \\
\end{array}$ & +0 & $\begin{array}{r}+0,89 \\
\end{array}$ & $+0,80$ & $\begin{array}{r}0,90 \\
+\end{array}$ \\
\hline 12 & $+1,60$ & $+1,38$ & $+1,30$ & $+1,17$ & $\begin{array}{r}+0.9 \\
\end{array}$ \\
\hline 13 & $+1,9$ & +1 & $+1,47$ & $+1,34$ & $+0,9$ \\
\hline 14 & $+2,25$ & $+1,79$ & $+1,69$ & $+1,54$ & $+0,9$ \\
\hline 15 & $+2,70$ & $+2,15$ & $-2,02$ & $+1,82$ & $+0,99$ \\
\hline
\end{tabular}

Die Ablesungen erfolgten bis 10 viertelstündlich, bis 12 nach je 3 Stunden, dann nach je 24 Stunden.

Tabelle der Gangunterschiede bei der Denitrierung von Ramie mit 10,55 Proz. N.

\begin{tabular}{r|c|c|c|c|c}
\hline & $435 \mu \mu$ & $546 \mu \mu$ & $579 \mu \mu$ & $650 \mu \mu$ & $\nu$ \\
\hline 1 & $+0,35$ & $+0,32$ & $+0,32$ & $+0,31$ & $+0,75$ \\
2 & $+0,46$ & $+0,41$ & $+0,40$ & $+0,38$ & $+0,76$ \\
3 & $+0,57$ & $+0,53$ & $+0,52$ & $+0,50$ & $+0,77$ \\
4 & $+0,75$ & $+0,66$ & $+0,64$ & $+0,58$ & $+0,81$ \\
5 & $+0,88$ & $+0,75$ & $+0,73$ & $+0,65$ & $+0,87$ \\
6 & +1 & $+0,78$ & $+0,77$ & $+0,73$ & $+0,89$ \\
7 & $+1,10$ & $+0,92$ & $+0,90$ & $+0,81$ & $+0,90$ \\
8 & $+1,21$ & $+1,05$ & $+0,95$ & $+0,9$ & $+0,90$ \\
9 & $+1,31$ & $+1,20$ & $+1,12$ & +1 & $+0,89$ \\
10 & $+1,44$ & $+1,29$ & $+1,20$ & $+1,08$ & $+0,91$ \\
11 & +150 & $+1,34$ & $+1,25$ & $+1,16$ & $+0,93$ \\
12 & $+1,60$ & $+1,38$ & $+1,30$ & $+1,17$ & $+0,92$ \\
13 & $+1,95$ & $+1,56$ & $+1,50$ & $+1,38$ & $+0,95$ \\
14 & $+2,25$ & $+1,84$ & $+1,72$ & $+1,52$ & $+0,97$ \\
15 & $+2,58$ & $+2,02$ & $+1,91$ & $+1,70$ & +1
\end{tabular}

Die Ablesungen erfolgten bis 7 viertelstündlich, bis 11 halbstündlich, bis 13 nach je 3 Stunden, zuletzt nach je 12 Stunden.

\section{IV.}

\section{Orientierung der Indexellipsoide.}

Zur Charakterisierung der Doppelbrechung ist es zweckmäBig, die Lage der Indexellipsoide zu bestimmen. Bei der reinen Ramie liegt die kleinste Achse des Ellipsoides radial, die mittlere tagential und die größte in der Längsrichtung der Faser. Da die Doppelbrechung des Querschnittes am geringsten ist, müssen die optischen Achsen so liegen, dab ihr spitzer 
Winkel durch die längste Achse des Ellipsoids halbiert wird.

Bei der Probe mit 13,16 Proz. N liegt dagegen die größte Achse der Indikatrix radial, die mittlere tangential, während die kleinste in die Längsrichtung der Faser fällt. Der spitze Winkel der optischen Achsen wird in diesen Fall von der kürzesten Achse des Ellipsoides halbiert.

Die Faser der reinen Ramie hat also dasselbe Indexellipsoid wie ein optisch zweiachsiger positiver Kristall, whihrend die hocbnitrierte das eines optisch zweiachsigen negativen Kristalles a $u$ fwe ist.

\section{V.}

\section{Akzidentelle Doppelbrechung.}

Bis jetzt war nur von der Doppelbrechung die Rede, die durch die Struktur bedingt ist. Nun beobachtete V. v. Ebne ${ }^{11}$ ), „daß an Bast fasern von Hanf und Lein im imbibierten $\mathrm{Zu}$ stand schon bei einer mäBigen Dehnung die Farbe steigt". Auch bei der reinen Ramie wurde die vorhandene positive Doppelbrechung durch die akzidentelle, welche durch die Dehnung hervorgerufen wurde, vermehrt. Bei den Proben mit 10,55 Proz. $\mathrm{N}$ und mit 11,88 Proz. $\mathrm{N}$ stieg in gleicher Weise die Interferenzfarbe wie bei reiner Ramie. Bei der Probe mit 13,16 Proz. $\mathrm{N}$ sank die Interferenzfarbe oder, mit anderen Worten, die vorhandene negative Doppelbrechung wurde durch die hinzukommende positive akzidentelle Doppelbrechung geschwächt. Die akzidentelle Anisotropie änderte den Betrag der bereits vorhandenen bis zum Reißen der Faser um etwa ein $1 / 20 \lambda$.

Ein'e Dehnung der Faser ruft folglich positive akzidentelle. Doppel. brechung hervor, gleichgültig, ob reine oder nitrierte Ramie vorliegt.

VI.

\section{Dichroismus.}

Durch die Untersuchungen von H. Ambron $n^{12}$ ) ist dargetan, dab die Anisotropie der Zellmembranen auch auf die Lage des $\mathrm{Ab}$ sorptionsellipsoides von EinfluB ist, wenn die

11) V. v. Ebner, Untersuchungen über die Aniisotropie organisierter Substanzen (1882), 212.

12) H. A mbronn, Ueber Pleochroismus pflanzlicher und tierischer Fasern, die mit Gold - und Silbersalzen gefärbt sind; Bet. đ. Kgl. Sächs. Ges. d. Wiss. za Leipzig, math.-phys. Klasse 48,613 ff. (1896).
Fasern in geeigneter Weise gefärbt werden. Nach den bereits mitgeteilten Beobachtungen über die Umlagerung der Achsen des Indexellipsoides durch die Nitrierung war es von Interesse zu untersuchen, ob bei diesem Vorgang, der eine so wesentliche Aenderung im optischen Verhalten der Faser bewirkt, auch eine entsprechende Umlagerung der Achsen des Absorptionsellipsoides einträte.

Leider läßt sich die in der botanischen Histologie viel benutzte Färbung mit Chlorzinkjodlösung zu diesem Zweck nicht verwenden, da die charakteristische Reaktion sich nur bei reiner Zellulose zeigt. Dagegen gelangen die Färbungen mit alkoholischer Kongorotlösung, Indigo und neutralem Methylenblau. Es sind die damit gefärbten Fasern am dunkelsten, wenn die Faserrichtung mit der Polarisationsebene des Nikols gekreuzt ist, am hellsten aber nach einer Drehung um 90\%, einerlei, ob reine Ramie oder eine der Nitrierungsstufen vorliegt. Auch bei Färbungen mit Goldchlorid oder Silbernitrat zeigte sich derselbe Dichroismus wie bei der rẻinen Faser.

Die später zu erwähnenden Erscheinungen der verschiedenen. Absorption im ultravioletten Licht von $280 \mu \mu$ kann man auch als Dichroismus auffassen; die nitrierte Faser verhält sich bei dieser Wellenlänge so, als ob sie intensiv gefärbt wäre. Hier wird aber die größere Lichtmenge durchgelassen, wenn Faserachse und Polarisationsebene gekreuzt liegen, und die stärkste Absorption erfolgt, wenn beide Richtungen parallel sind. Diese Verschiedenheit in dem Absorptionsverhältnis bei gefärbten und ungefärbten Fasem rührt wohl daher, daß in dem einen Falle die Absorption auf der molekularen Struktur der Faser selbst beruht, während sie bei den künstlichen Färbungen auf die Natur des eingelagerten Farbstoffs zurückzuführen ist.

Es hat sich also bei allen Färbungen das interessante Resultat ergeben, daß durch die Nitrierung keine. Umlagerung der Achsen des Absorptionsellipsoides bewirkt wird; auch die Fasern mit dem höchsten Stickstoffgehalt, die also in bezug auf die Längsrichtung sich als stark negativ erweisen, lassen bei allen diesen Färbungen dieselben Absorptionsverhältnisse erkennen, wie sie bei der reinen Ramie beobachtet werden. Von besonderem Interesse ist noch, dab diejenigen Nitrierungsstufen, bei denen für eine Wellenlänge Isotropie besteht und für die anderen nur sehr schwache Doppelbrechung von entgegengesetztem Vorzeichen vorliegt, eben- 
falls denselben starken Dichroismus zeigen, wie die reine Ramie und die höchste Nitrierungsstufe.

Wenn die Ansicht $H$. Ambronn's richtig ist, dab der Dichroismus durch die gleichmäBige Orientierung der an sich räumlich und optisch anisotropen Farbstoffteilchen entsteht, so mup also bei der nitrierten Ramie die Einlagerung in derselben Orientierung stattfinden, wie in der reinen; $d . h$. die intermizellaren Raume, in denen die Einlagerung erfolgt, mussen im wesentlichen dieselben bleiben, auch wenn der chemische und physikalische Chatakter der einzelnen Mizelle, selbst stark geăndert wird.

VII.

Untersuchungen im U. V.-Licht.

Es war nun noch van Interesse, zu untersuchen, ob auch bei Belenchtung mit ultraviolettem Licht Unterschiede zwischen reinen und nitrierten Fasern sich ergaben. Zunächst wurde mittels einer $Z$ e iss'schen U. V.-Filterlampe nach $H$. Lehmann, wie sie bereits zut Untersuchung der Lumineszenzerscheinungen von Mineralien benutzt worden ist ${ }^{13}$ ), sowohl die reine Ramie wie auch die verschiedenen Nitrierungsstufen im ultravioletten Lichte vor 300 bis $400 \mu \mu$ geprüft. Es ergab sich, daB alle Fasern eine deutliche Lumineszenzstrahlung aussenden, die bei der reinen Ramie am intensivsten ist, bei den nitrierten Fasern aber mit steigendem Stickstoffgehait in ihrer Intensität allmählich abnimmt. Bemerkenswert ist aber, dah der Polarisatioriszustand des ausgestrahiten Lichtes in beiden Fällen der gleiche ist: die Fasern leuchten an hellsten auf, wenn die Polarisationsebene des zur Beobachtung benutzten Analysators senkrecht zur Längsachse steht. Man hätte vielleicht erwarten können, daß die vitrierten Fasern gerade die entgegen: gesetzte Polarisation wie die reinen Fasern zeigen würden; die Beobachtung hat aber mit Sicherheit ergeben, daß nach dieser Richtung kein wesentlicher Unterschied besteht.

Ferner konnte ich durch das freundliche Entgegenkommen des Herrn Dr. A. Köhler, dem ich hierfür auch an dieser Stelle meinen verbindlichsten Dank ausspreche, die verschicdene Absorption der Fasern im ultravioletten Licht von $280 \mu \mu$ untersuchen. Die Absorption der reinen Faser ist sehr gering und zeigt beim

13) Vgl, E. Engelhardt, Lumineszenzerschelnungen der Mineralien im U. V.- Licht (Inaug.-Dissert., Jena 1912).
Drehen über einem geelgneten Polarisator keine Verschiedenheit. Schon die niedrigsten Nitrierungsstufen ergeben dagegen eine beträchtliche Absorption, die aber bei böherem Stickstoffgehalt nicht mehr wesentlich stärker wird. Bei dem Drehen der nitrierten Fasern über dem Polarisator zeigt sich jedoch eine deutliche Verschiedenheit in der Weise, dab die Pasern am hellsten erscheinen, wenn Polarisationsebene und Faserachse gekreuzt sind.

Die im vorstehenden wiedergegebenen $\mathrm{Be}$ obachtungsresultate lassen sich wohl am besten auf Grund der $\mathrm{N}$ äg e li'schen Mizellarhypothese verständlich machen. Macht man nämlich die Anuahme, dab die Mizellen an sich schon optisch anisotrop sind und daß durch die Nitrierung der optische Charakter des einzelnen Mizells ungekehrt wird, die Gesamtorientierung aber im wesentlichen erhalten bleibt, so finden die beobachteten Erscheinungen eine ganz ungezwungene Erklärung. Man könnte dem. nach die Umwandlung der Zellulosefaserin eine nitrierte Faser auch als eine Art von Pseudomorphose - - im Sinne der Mineralogie - von Nitrozellulose n a ch Z ellulose a uffassen; das Zellulosemizell wird in ein Nitrozellulosemizell ungewandeit, aber die Orientierting und somit die äuf̧ere Gestalt des ganzen Systems bleibt erhalten. Nimmt man, wie schon gesagt, an, dafs das Vorzeichen der Doppelbrechung bei beiden entgegengesetzt ist, so wird auch sofort verständlich, warum bei allmählicher Nitrierung und Denitrierung der Charakter der Anisotropie in den garzen Fasern eine Umkehrung erfahren muls.

Etwas ganz Analoges tritt bei manchen Kristallen, besonders aus der Gruppe der Zeolithe, in Erscheinung, bei denen nach Beobachtungen von $F$. Rinn $\mathrm{e}^{14}$ ) und anderen durch bestimmte Einwirkungen ein „kristallographisch-chemischer Ab- und Umbau" stattfindet. Auch bei diesen Kristallen wird unter Beibehaltung der äuBeren Form eine starke Veränderung des optischen Verbaltens und in manchen Fällen auch eine Umkehr des Charakters der Doppelbrechung beobachtet. Ebenso wie in solchen Fallen des kristallographisch-chemischen Umbaues manchmal eine Umkehrbarkeit des Vorganges beobachtet werden konnte, so läBt sich auch, wie

14) F. Rinne, Kristallographisch-chemischer Abund Umbau, insbesondere von Zeolithen; Fortschritte der Mineralogie, Kristallographie und Petrographie $\mathbf{3}$, 159 (1913). 
gezeigt wurde, die nitrierte Paser wieder in den früheren reinen Zellulosezustand überführen.

Ferner zeigen auch die Mischkristaile aus Blei- und Strontiumdithionat eine gewisse Aehnlichkeit im optischen Verhalten. Das Bleisalz ist optisch positiv doppelbrechend, das Strontiumsaiz dagegen negativ. Man kann nun, wie H. Ambron ${ }^{15}$ ) gezeigt hat, sehr gut verfolgen, wie in den Mischlrristallen bei steigendem Gehalt an Strontiumsalz die positive Doppelbrechung immer schwächer wird, schließlich ganz verschwindet und darauf die negative eintritt. Dabei sind die starken Veränderungen im Gange der Dispersion der Doppelbrechang ganz denen entsprechend, die wir bei der $\mathrm{Ni}$ trierung und der Denitrierung der Fasern beobachten konnten.

15) H. Ambronn, Ueber die Dispersion der Doppelbrechung in den Mischkristallen von Strontiurnund Bleidithionat; Zeitschr. f. Kristallographie usw. 52, $48-57$ (1913).
Als ein wichtiges Ergebnis der Untersuchung ist aber vor allem her. vorzuheben, dab zwischen dem Stick. st offgehalt und den optischen Eigenschaften der Faser eine ganz bestimmte Beziehung besteht. Man kann ohne $Z$ weifel durch die sachgemäß ausgeführte mikroskopische Untersuchung schon an einzelnen Fasern sofort erkennen, ob eine niedrigere oder höhere Nitrierungsstufe vorliegt. Ferner läßt sich auf diese Weise auch rasch feststellen, ob in. einer zu untersuchenden Probe optisch verschieden wirkende Fasern vorhanden sind, ob es sich also um ein gleichmäßig oder ungleichmäBig nitriertes Material handelt. Es darf deshalb wohl die Erwartung atisge. sprochen werden, dab sich die Prüfung der nitrierten Pasern im Polarisationsmikroskop auch für die Praxis in der Nitrozelluloseindustrie von grobem Vorteil erweisen werde.

Jena. August 1913.

\section{Untersuchung über Natur und Eigenschaften von Hevea-Latex. ${ }^{\text {') }}$}

Von Clayton Beadle und Henry P. Stevens.

(Abdruck aus den Orlginal-Mittellungen des 8. internationalen Kongresses für angewandte Chemle.)

1. Teil.

\section{Physikalische Eigenschaften und Koagulation} oon Hevea-Latex.

Die von uns berichteten Untersuchungen wurden teils von dem einen von uns mit frischem Latex im Osten, zum anderen Teil an nach Zusatz von Konservierungsmitteln mitgebrachtem Latex angestellt. Von den früheren Arbeiten über diesen Gegenstand ist die wichtigste die von V. Henria) in Europa mit konservierten Latex ausgeführte Untersuchung. jedoch ist die Konservierungsmethode nicht angegeben. Wir erfahren nur, daB der.Latex eine schwach alkalische Reaktion aufwies. Nun zeigt aber Latex, wie er aus dem Baum austritt, gelegentlich eine schwach alkalische Reaktion, sobald neutrales Lackmuspapier an den eben aus einem frischen Einhieb austretenden Tropfen gedrückt wird. Indessen ist die Reaktion nicht" unveränderiich alkalisch, sie kann auch neutral oder sogar schwach satter sein infolge der Vermischung mit Saft aus den

1) Uebersetzung von $\mathrm{H}$. Brehm (Dresden).

2) V. Henri, Le Caoutchoue et la Guttapercha 1906, 510; 1907, 1066; 1908, 2405. Ferner Le Latex (Th. Bosquet, Paris.)
Gefagen, die natürlich zur selben Zeit durchtrennt werden, wie die Latex führenden Gefäbstrănge. $\mathrm{Zu}$ der Zeit, wenn der eingesammelte Latex in der Zentralstation einer Plantage abgeliefert wird, ist derselbe unbedingt schwach sauer gegenüber Lackmus. Phenolphthalein kann als Indikator zur Bestimmung der Azidität von Latex verwendet werden, wenn man mit n/100 Alkalilösung titriert.

Man hat häufig Formalin zur Konservierung von latex verwendet, wenn letzterer nach Europa transportiert werden sollte; jedoch ist die dazu erforderliche Menge nicht unbeträchtlich, nämlich. ungefähr 2 bis 3 Proz. in Form einer aum Latexvolumen passenden Verdünnung. Die Konservierung eines solchen Latex ist jedoch eine mehr scheinbare als latsächliche. Eine mikroskopische Untersuchung ergibt, dab der mit Formalin behandelte Latex einer beginnenden Koagulation unterlegen hat, in dem der grölere Tell aus Aggregaten von teils kleinen, teils viel größeren Kügelchien zusammengesetzt ist, und daß der Anteil an in B rown'scher Molekularbewegung befindlichen freien Kügelchen verhälínismäßig gering ist, oder daB solche überhaupt völlig fehlen. Ein 